\title{
Hydronic Optimization of Hybrid Heat Production Systems: a Methodology Based on Base Circuits
}

\author{
Van Riet Freek ${ }^{1}$, Vandenbulcke Roel ${ }^{2}$, Cleiren Jonas ${ }^{2}$, Verhaert Ivan ${ }^{1}$ \\ ${ }^{1}$ EMIB research group, University of Antwerp, Antwerp, Belgium \\ ${ }^{2}$ Hysopt NV, Antwerp, Belgium
}

\begin{abstract}
Heat is responsible for over $50 \%$ of the energy end use in Europe. Hybrid heating systems based on sustainable technologies such as cogeneration and heat pumps have the potential to increase energy efficiency and/or share of renewable energy in the energy supply for heat. In order to achieve these saving potential, hydronic integration is critical. To optimize the design of this integration, a simulation-based design methodology is proposed. The methodology is characterized by a Base Circuit approach, which is incorporated in the design and simulation software Hysopt.

A representative, but simplified case study is implemented in the software to illustrate the optimization potential of hydronic integration for hybrid heating purposes. Differences in primary energy savings of up to 20 percentage points were reported, indicating the importance of hydronic optimization.
\end{abstract}

\section{Introduction}

\section{Problem statement}

Over the past decades, the integration of sustainable heat production technologies in buildings has been increasing (Zimny et al., 2015; Verhaert et al., 2019). This integration is, amongst others, facilitated by the trend towards collective heating, as the used technologies are subject to economies of scale (Nielsen et al., 2016; Gimelli et al., 2017). For large buildings, two technologies in particular are wide-spread: Internal Combustion Engine-based Combined Heat and Power (ICE-CHP) and Ground-Coupled Heat Pumps (GCHP).

The disadvantage of both technologies is their capital cost. If one of these two technologies is implemented in a building, it is therefore often combined with an inexpensive auxiliary heater to be able to reduce the size of the expensive technology (Ren et al. (2008); Hackel and Pertzborn (2011); Di Perna et al. (2015)).

Such hybrid heat production systems are highly complex to design. While great efforts have been made to select and size the different heat production devices (Ren et al., 2008; Barbieri et al., 2014; Fuentes-Cortés et al., 2015, e.g.), the hydronic aspect of the design has only recently been subject to scientific research. The available literature reveals a substantial effect of it on the performance of the production system, though. Indeed, for a hybrid production system with an ICE-CHP different hydronic concepts were compared (serial, parallel and shunt connections between CHP and boiler) and showed a difference of up to 10 percentage points of relative primary energy savings (Van Riet et al. (2018b, 2019)). For heat pumps the differences were found to be even more expressed (up to 20 percentage points difference, Van Riet et al. (2018c)).

Upper research was performed with a script-based simulation environment, strictly for academic use. While some effort has been made to decrease scripting time of the hydronic distribution system in this environment (Van Riet et al. (2018a)), it remains labour-intensive to use.

The susceptibility of hybrid heat production systems to the hydronic design, and its complexity that installers, engineer offices and manufacturers have to deal with, highlight the need for a methodology which is more accessible for its users.

\section{Scope and outline}

This paper proposes a methodology to design hybrid heat production systems consisting of a 'principal' installation (ICE-CHP or GCHP) combined with an auxiliary boiler. The methodology is based on the so-called Base Circuits, developed by Vandenbulcke et al. (2012) and implemented in the software Hysopt. This software focusses on user-friendliness, thereby providing a design tool at the level of installers, engineering offices and manufacturers. The paper aims to illustrate the Base Circuit Methodology applied on hybrid heat production systems.

In order to do so, the general methodology is explained in the next section. Then, the case study and corresponding boundary conditions of the design are described. Finally, the hydronic design concepts are explained and evaluated based on the proposed methodology. 


\section{General methodology}

While a variety of drawing (CAD) software solutions are available for HVAC designs, a huge gap exists when it comes to calculation and simulation. Hysopt develops and markets design- and simulation-software for designers within engineering offices, installers, ESCO's and building facility managers.

As illustrated in Figure 1, the innovative 'Base Circuit' design methodology links pre-programmed Base Circuits (BC) to each other in order to create a mathematical model of the HVAC or district heating system. Whereas most simulation software consider the HVAC system as a strictly thermal problem, the Hysopt software enables both thermal and hydraulic system calculation and analyses due to a combined thermal and hydraulic equation solver based on Newton-Raphson iteration.

During the modelling, conceptual design faults are avoided thanks to a 'system check' that aims to guarantee the design quality at an early stage. Therefore a system conceptual typology is embedded into each BC so that the software basically 'knows' what is built. E.g. incorrect positions of pumps will generate a warning for the user.

After setting the required thermal loads and temperature levels for end units, the design flow rates are calculated and, based on that, suitable pipes are selected. In a next step, advanced iterative optimisation algorithms are used to automatically select system components such as pumps, control valves, balancing valves and radiator characteristics. This provides an alternative for the conventional labour-intensive manual calculations and inaccurate rules of thumb. The optimization algorithm takes minimal control valve authority into account and so improves the controllability of the system (speed, stability, accuracy).

The software allows to design on both schematic diagrams (P\&ID) and floor plans (dxf import file). P\&IDs are mainly recommended for more complex hydraulic sections of the installation such as a boiler room and substations. Floor plans are scanned to calculate pipe lengths and bends automatically. In order to keep a visual overview, different canvasses (parts of the whole system) can be viewed separately while the nodes of the BCs remain connected.

In general, Hysopt aims to predict system performances in a design phase, taking the installation's dynamic partial load behaviour into account. The interactions between the building and its HVAC system can be analysed based on variable weather data and temperature set-points of the rooms. Advanced DDC techniques and control strategies can then be implemented in the virtual HVAC system using generic control blocks and freely programmable control logics.
After the dynamic simulation, the user can extract all variables (flow rate, pressure, temperature, valve position, control signals, etc.) at all possible locations in the installation, which is visualised by the software (see e.g. Figure 4). The energy consumption of the heating and cooling production assets (boiler, heat pump, CHP, chiller, etc.) can also be quantified alongside the auxiliary energy required for pumps and fans.

Due to the relative small time constants of HVAC system processes and DDC controls, the dynamic system simulations run on a $30 \mathrm{~s}$ step size. To cope with the corresponding computational challenges, all calculations run on Amazon cloud servers which are optimized for calculation speed and are directly accessible via the Hysopt web interface.

Compared to other modelling software which can be used for hydronic system simulations (e.g. Modelica, Engineering Equation Solver) the hydraulic solver within the Hysopt software is tailored for the specific hydraulic equation sets encountered in large heating and cooling systems. Doing so, simulations are performed a magnitude faster compared to the known and more general-purpose solvers. Moreover, all cloud servers are optimized for calculation speed and allow the designer to run different system alternatives and sensitivity analyses simultaneously on different CPUs. Besides, it should be noted that the Hysopt software is not only a simulation software but also automates design calculations. Accordingly, simulations are performed on properly designed systems to return accurate predictions and conclusions. In design processes, the overall aim is eventually to bring system simulation to real application. Therefore the software usability is top priority and its interface focusses on designers in engineering offices and installers, rather than pure academics.

The description of thermal and hydraulic models of the BCs that are implemented in the software can be found in Vandebulcke (2013) and Van Riet et al. (2016).

\section{Boundary conditions}

\section{Case description}

From literature and experience, it is known that thermal efficiency of a boiler and CHP increases with lower inlet temperatures, whereas the coefficient of performance (COP) of a heat pump is high at a low outlet temperature.

To illustrate both the influence of the hydronic design and the potential of the methodologic approach discussed in the previous section, a case study is selected with two heating circuits, each at a different temperature level (Figure 2). Designs that are more complex are also possible, but for the purpose of a comprehensive demonstration of the methodology, 


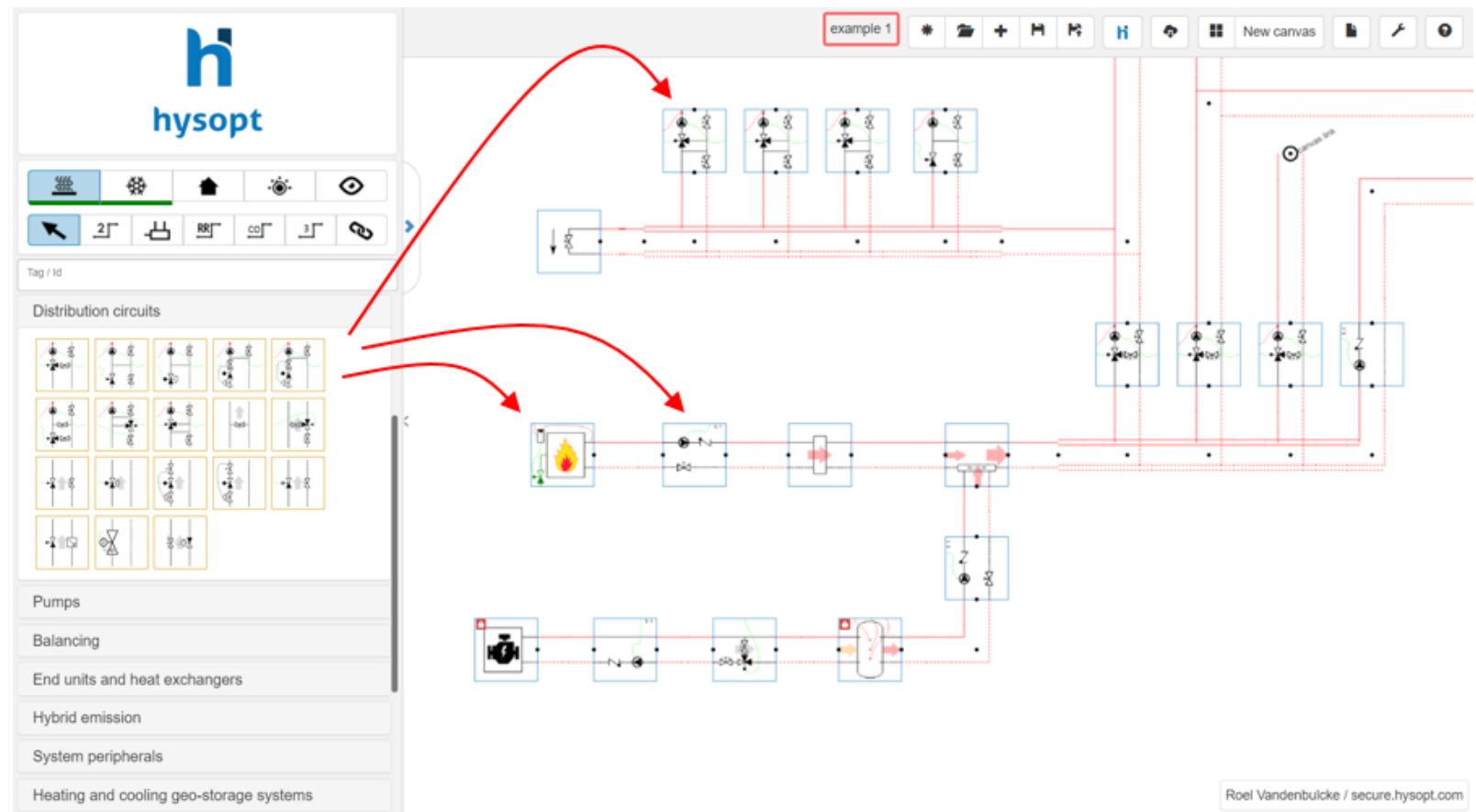

Figure 1: Illustration of the Hysopt software where the installation is assembled from hydraulic Base Circuits with internal intelligence using an intuitive drag \& drop interface.

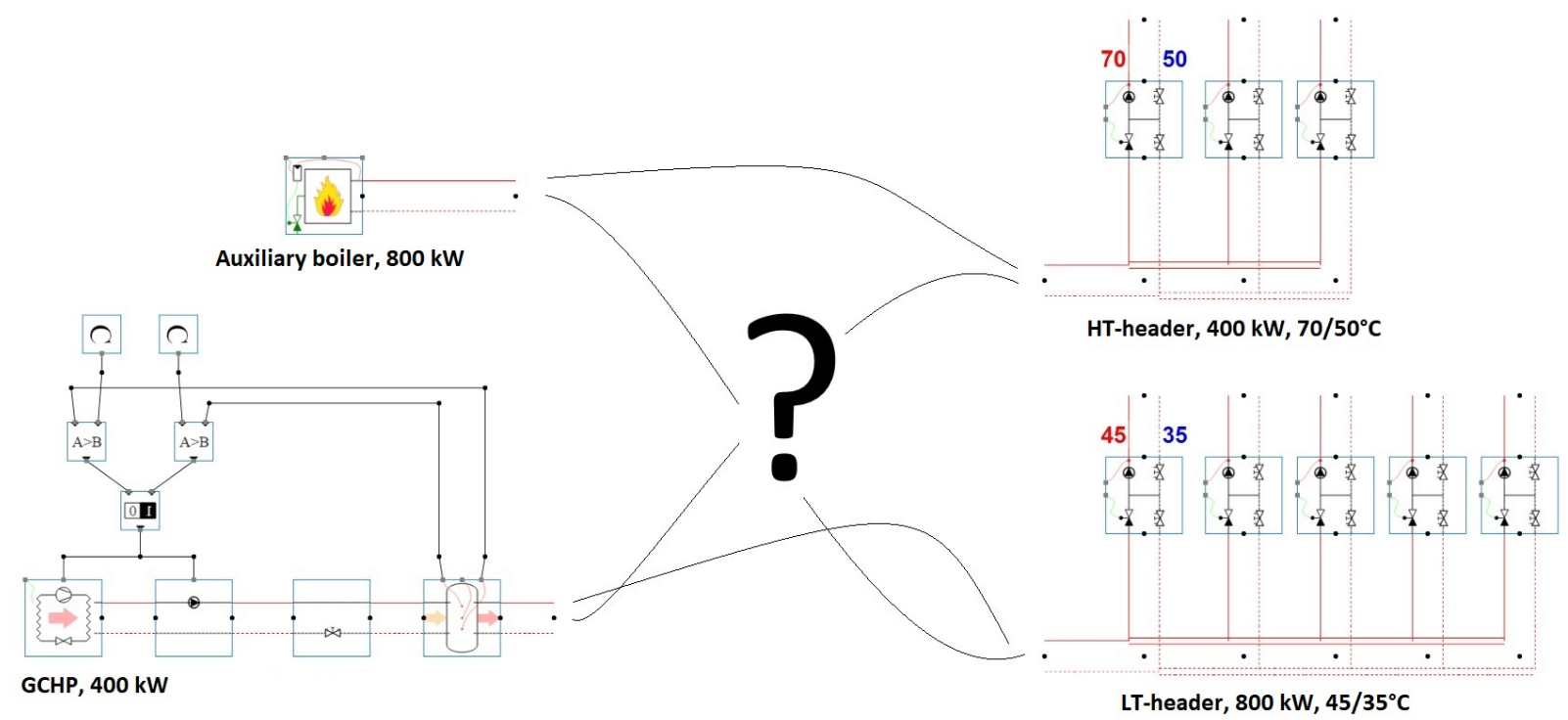

Figure 2: Schematic overview of case study and research question: "How to connect the different heat producers to the different end-uses?". Only a GCHP is shown as principal heat producer. 
it is chosen to simplify this. The high temperature (HT) circuit has a heat demand of $400 \mathrm{~kW}$ and is designed to work at a regime of $70 / 50{ }^{\circ} \mathrm{C}$, at design conditions. The low temperature (LT) circuit has a demand of $800 \mathrm{~kW}$ at $45 / 35{ }^{\circ} \mathrm{C}$, also at design conditions.

These boundary conditions are typical for buildings with floor heating and domestic hot water, air-handling units and radiators, or a combination of previous illustrations.

The total heat demand of $1200 \mathrm{~kW}$ is delivered by a hybrid heating production system, existing out of condensing boiler and a principal heat producer: either a ground-coupled heat pump (GCHP) or cogeneration unit (CHP). Typically, the GCHP and CHP are not sized on peak load, but on a base load, as discussed in the introduction. Based on the methodology of annual load duration curve, a base load of $400 \mathrm{~kW}$ is chosen to be the size of heat pump and of $120 \mathrm{~kW}$ of the CHP. The condensing boiler is sized to cover the peak loads. With this information, Figure 2 illustrates the remaining design question, namely: "How to connect those different heaters to the different end-uses?" The figure shows an example of only a GCHP as principal heat producer.

\section{Performance criteria}

To discuss the results of the simulations in the next section (more specifically Table 1), the data is summarised into Key Performance Indicators (KPI's) of the production system:

- $\eta$ : the principal heat producer is evaluated by its yearly thermal efficiency $\left(\eta_{t h}\right.$, which is the SPF for the GCHP) and, for the CHP, by its yearly electrical efficiency $\eta_{e l}$. The auxiliary heater is evaluated by its thermal efficiency, $\eta_{b o i l e r}$.

- 'share': the percentage of the heat produced by the principal heat producer of the total consumed thermal energy by the building. The remaining share $\left(100-^{\prime}\right.$ share $\left.{ }^{\prime}\right)$ is, obviously, produced by the auxiliary boiler. An hydronic circuit should be designed to maximise the share of the principal producer.

- RPES: the relative primary energy savings are calculated with a reference electrical and thermal efficiency of $37 \%$ and $90 \%$ (based on higher heating value), respectively, that represent the best available conventional heat and electricity production alternative, as discussed by Verhaert et al. (2016). This variable takes both the principal heat producer's and auxiliary boiler's performance into account.

- $t_{c y c}$ : the mean continuous operation time in hours between a start-up and shut-down of the principal heat producer. It quantifies the stability of operation: the higher the number the less maintenance costs can be expected. The princi- pal producer is also evaluated by its total operating time (hours).

- 'savings \%': the financial savings, relative to a reference electrical and thermal efficiency of $37 \%$ and $90 \%$, and assuming an electricity price of $0.11 € / \mathrm{kWh}$ and a gas price of $0.04 € / \mathrm{kWh}$.

\section{Hydronic design: concepts and evalua- tion}

This section elaborates on the working of different hydronic designs and their performance. In the first subsection, the designs with a GCHP ('GCHP 1', 'GCHP 2', 'GCHP 3', 'GCHP 4', 'GCHP 5', 'GCHP 6') are discussed and in the second, those with a CHP ('CHP 1', 'CHP 2', 'CHP 3'). All concepts are schematically shown as draw in Hysopt in Figure 3 for the GCHP and Figure 5 for the CHP, and the results are given in Table 1 . The considered hydronic concepts are both existing concepts applied in practice, as well as improved ones proposed by the authors of this paper.

\section{Ground-Coupled Heat Pumps}

Concept 'GCHP 1' and ' GCHP 2'

For both concepts 'GCHP 1' and 'GCHP 2', the boiler is implemented in parallel with the heat pump. The temperature of the return water going towards the boiler is the mean of the return temperatures of the HT- and LT-circuit, which is, at design conditions, equal to $42{ }^{\circ} \mathrm{C}$. This low temperature results in a high boiler efficiency of $94 \%$ for both design concepts.

While an acceptable SPF can be achieved, the share of heat produced by the GCHP is limited for 'GCHP 1': $19 \%$. This can be explained by the parallel flow towards the boiler. Indeed, this parallel flow is allowed by the opening of an open-closed two way valve (see Figure 3) when the boiler is on. Therefore, the discharging flow of the storage tank is decreased, which can be seen in Figure 4. Since the GCHP's operation is based on the state of charge of this tank, the lower discharging rate can cause a shut-down of the GCHP.

Such disadvantage of the parallel configuration can be partially solved by replacing the open-closed valve by a modulating valve, which is the case for concept 'GCHP 2'. It enables to decrease the flow rate towards the boiler, thereby increasing the flow rate to discharge the tank. However, results (Table 1) show that an increase in the share of the GCHP, and, correspondingly the RPES, of only 1 percentage point is possible. This contradicts to previous research (Van Riet et al., 2018c), which showed a higher level of improvement of a modulating valve. Other control strategies of that valve were considered, which do not allow to compare the results exactly. Hence, it is 


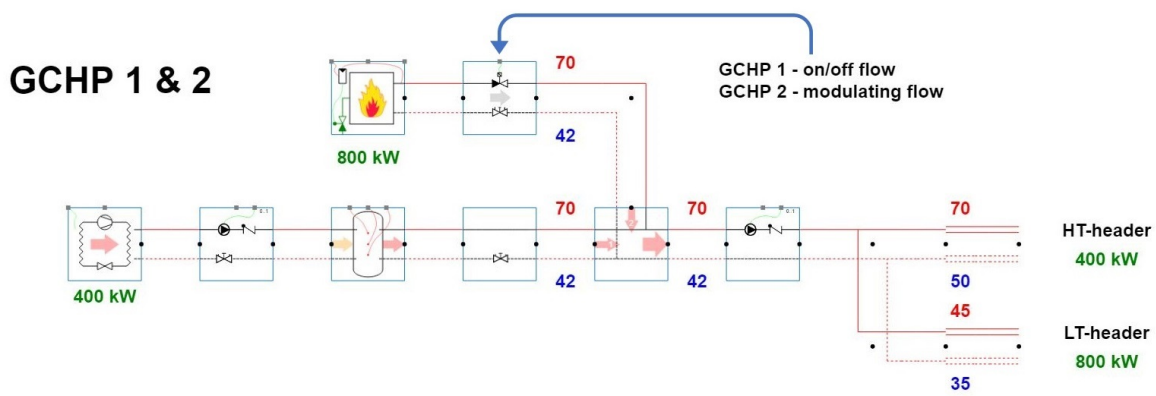

\section{GCHP 3}
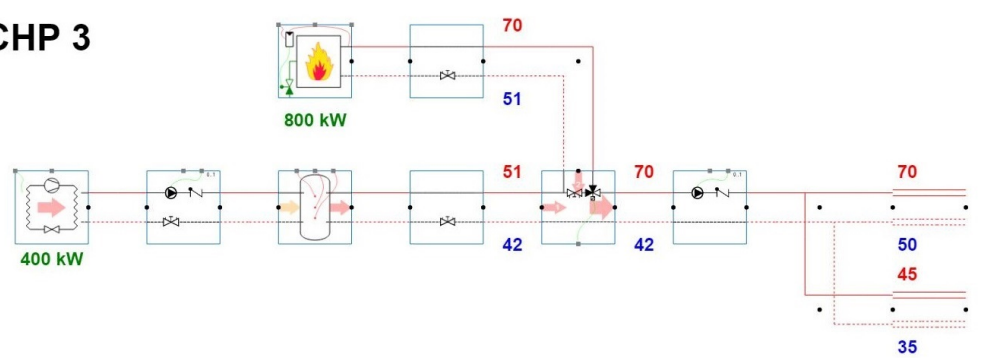

$400 \mathrm{~kW}$

LT-header $800 \mathrm{~kW}$

GCHP 4

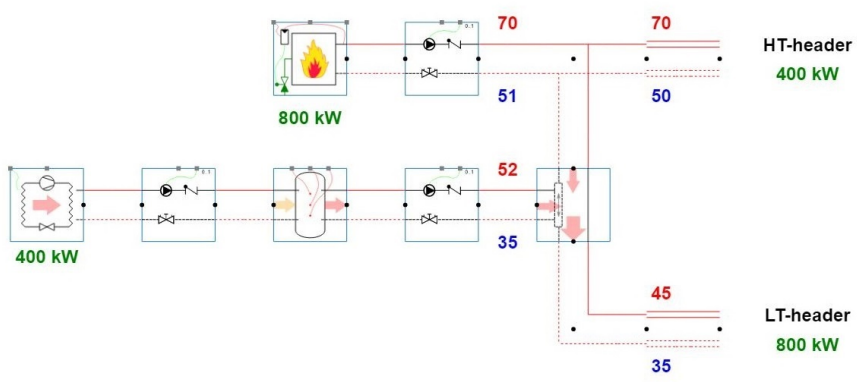

GCHP 5

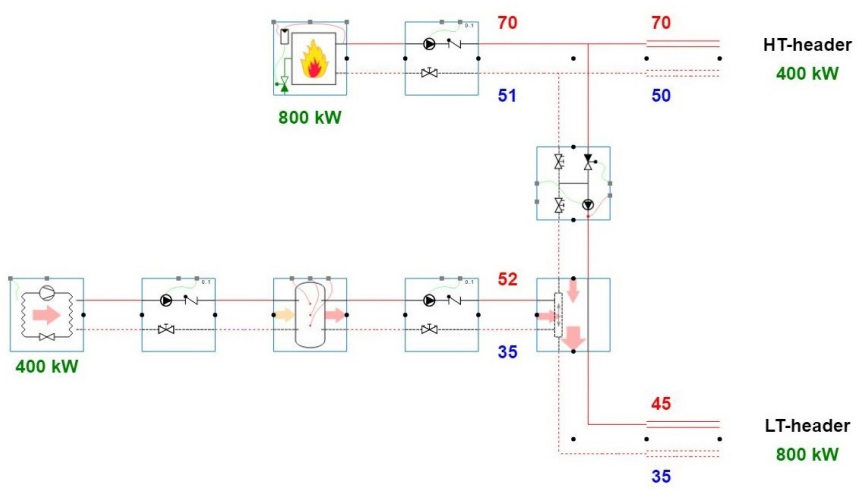

GCHP 6

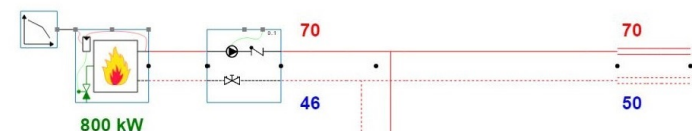

HT-heade $400 \mathrm{~kW}$

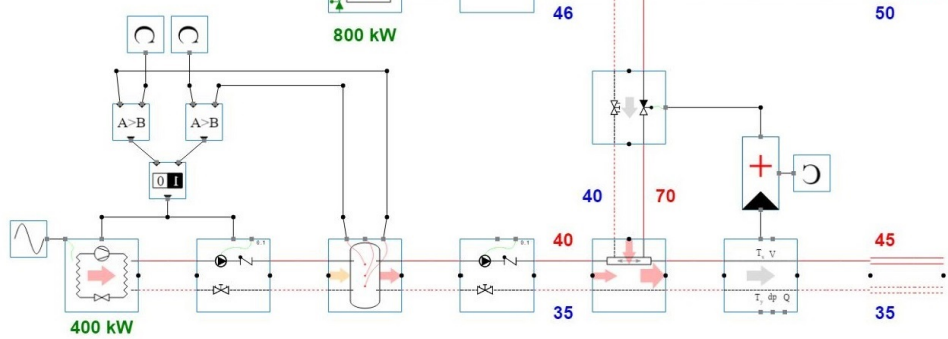

Figure 3: Hydronic concepts with a GCHP as principal heat producer. 
Table 1: Results of the performance of all design concepts for both GCHP and CHP. The symbol '*' indicates that the units are expressed in \% for the CHP and are dimensionless for the GCHP results, i.e. $\eta_{\text {th }}$ for the GCHP is the Seasonal Performance Factor.

\begin{tabular}{|c|c|c|c|c|c|c|c|c|}
\hline Concept & $\eta_{t h}{ }^{*}$ & $\eta_{e l}[\%]$ & $\eta_{\text {boiler }}[\%]$ & share [\%] & RPES [\%] & operation [h] & $t_{c y c}$ & saving [\%] \\
\hline GCHP 1 & 3.81 & $/$ & 94 & 19 & 11 & 1215 & 0.3 & 5 \\
\hline GCHP 2 & 3.81 & $/$ & 94 & 20 & 12 & 1284 & 0.3 & 5 \\
\hline GCHP 3 & 3.87 & $/$ & 89 & 51 & 20 & 2772 & 0.4 & 13 \\
\hline GCHP 4 & 3.79 & $/$ & 91 & 29 & 13 & 1920 & 0.3 & 5 \\
\hline GCHP 5 & 3.76 & $/$ & 93 & 63 & 26 & 3576 & 0.5 & 20 \\
\hline GCHP 6 & 4.21 & $/$ & 92 & 64 & 31 & 3420 & 0.7 & 23 \\
\hline CHP 1 & 56 & 35 & 76 & 24 & 16 & 6098 & 0 & 23 \\
\hline CHP 2 & 56 & 36 & 93 & 28 & 18 & 6721 & 9.4 & 26 \\
\hline CHP 3 & 56 & 36 & 92 & 28 & 18 & 6754 & 10.4 & 25 \\
\hline
\end{tabular}
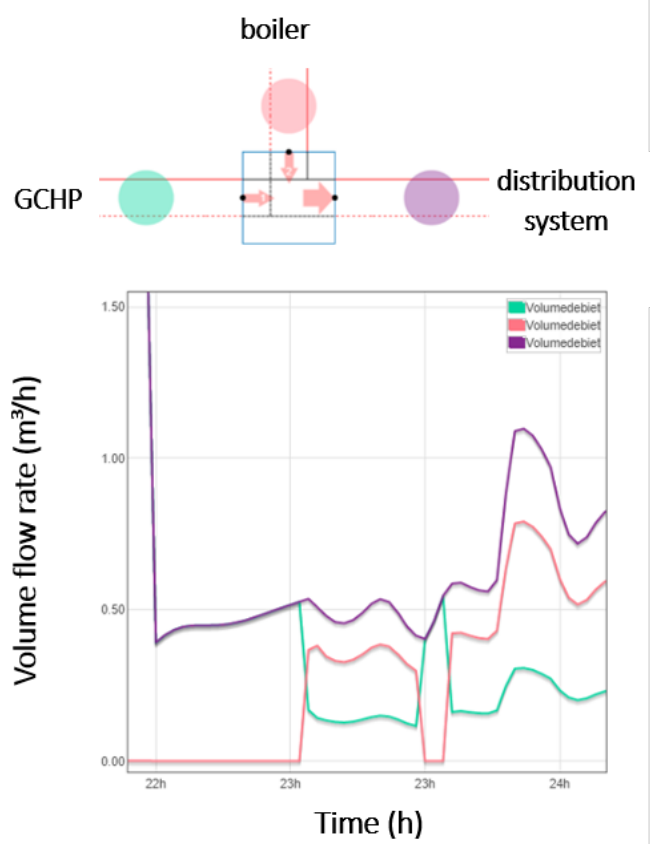

Figure 4: Decreased discharging rate of the storage tank, caused by the parallel flow towards the boiler. expected that the higher improvements of 'GCHP 2' are possible by optimising the control strategy.

Concept 'GCHP 3'

Another option to solve the disadvantage of 'GCHP 1 ' is to connect the boiler in series with the GCHP (Figure 3). By doing so, the complete flow rate of the distribution system is used to discharge the tank. The lower boiler efficiency (89\%), corresponding to the higher boiler inlet temperature, is compensated by the increased GCHP share (51\%) and SPF (3.89): RPES of $20 \%$ are possible. Obviously, this also results in higher financial savings (13\%).

Concept 'GCHP 4' and 'GCHP 5'

To increase the SPF of the GCHP, it can be implemented to produce heat for the LT-circuit only (return temperature of only $35^{\circ} \mathrm{C}$ at design conditions). In order to do so, the GCHP is connected in shunt of the LT-circuit in concept 'GCHP 4'. However, results reveal that the intended increase in $\mathrm{SPF}$ is not possible; it is even slightly lower (3.79) than for the concepts above. Also a lower GCHP share, RPES and financial savings can be seen in Table 1. The bypassing of water by the hydraulic separator, causing water to circulate in the GCHP circuit, can explain the results. Indeed, this is in accordance to previous research (Van Riet et al., 2018c).

While that same research examined another control strategy of the pump between hydraulic separator and storage tank to solve the bypassing, an alternative solution is proposed here by concept 'GCHP 5 '. By implementing a mixing valve in the LT-circuit to decrease the supply water temperature $\left(45^{\circ} \mathrm{C}\right.$ instead of $70^{\circ} \mathrm{C}$ ), the flow rate in the LT-distribution system is increased. As a consequence, less water is bypassed by the hydraulic separator, and the GCHP share, RPES and financial savings are increased up to $63 \%, 26 \%$ and $20 \%$, respectively. 
Note that concept 'GCHP 5' still results in a low SPF of 3.76. The dynamic simulations (not shown) revealed that at low demands of the LT-circuit -hence low flow rates- water is still bypassed by the hydraulic separated, resulting in higher temperatures for the GCHP. While outside the scope of this paper, a combination of concept 'GCHP 5' and the pump control discussed by Van Riet et al. (2018c) might increase the SPF.

\section{Concept 'GCHP 6',}

Another solution for this low SPF is proposed by concept 'GCHP 6': the position of the hydraulic separator is changed. More specifically, the boiler is connected in shunt on the supply pipe of the LT-circuit. This prevents circulation towards the GCHP. While an increase in GCHP share of only one percentage point is achieved, compared to the concept 'GCHP 5', the higher SPF (4.21) results in the highest possible RPES (31\%) and financial savings $(23 \%)$ of all GCHP designs. Also the stability of operation of the GCHP, expressed in a mean continuous operating time of $0.7 \mathrm{~h}$, seems to be the highest for this design.

\section{Internal Combustion Engine-based CHP}

For designs with an ICE-CHP, not all advanced concepts discussed in the previous section are likely to increase performance. The main reason for that is because the effect of temperature level on both the electrical and thermal efficiency of ICE-CHP device is limited. Therefore, only three different concepts are compared (Figure 5).

The characteristics of both concepts are analogue to those of 'GCHP 1' and 'GCHP 2'. Not surprisingly, similar effects can be see in Table 1. More specifically, concept 'CHP 2' is able to increase the CHP share, compared to concept 'CHP 1', and therefore also energetic and financial savings. Also the stability of operation of the CHP is improved substantially by considering a modulating two-way valve. The results of concept 'CHP 3' are similar to those of concept 'CHP 2': slightly lower financial savings $(<1$ percentage point) but a higher mean continuous operating time $(10.4 \mathrm{~h}$ instead of $9.4 \mathrm{~h})$. Except for the low boiler efficiency of concept 'CHP 1', these findings are in accordance with previous research (Van Riet et al., 2019). Again, another control strategy of the boiler can explain this difference.

\section{Concluding remarks}

This paper proposed a simulation-based methodology to design hybrid heat production systems. To illustrate the so-called Base-Circuit Methodology, it was applied on a case study.
For this particular case study, the following can be concluded:

- If a GCHP is implemented in the heat production system, the hydronics should be designed according to, what is introduced in this paper as, concept 'GCHP 6'. It allows Relative Primary Energy Savings and financial savings of, respectively, 20 and 18 percentage point higher, compared to the concept with the lowest performance (concept 'GCHP 1').

- For designs with a CHP, both concepts 'CHP 2' and 'CHP 3' show a similar, high performance. While 'CHP 2' can achieve slightly (less than one percentage points) higher relative financial savings, it requires a complex control strategy to do so. The choice of concept should therefore be based on the expected risk of failure of the complex control strategy.

These conclusions highlight the importance of hydronics: it should not be overlooked in design processes. The given conclusions cannot be generalised for other case studies, though, as the boundary conditions may differ. Hence, a comparison of design concepts should be made for each single case to achieve optimal hydronic performance. Since the proposed methodology proved to be successful to find the optima for the case studies considered in this paper, it is expected to do the same for others.

In conclusion, it can be said that this methodology can guide installers, design engineers and manufacturers in their pursuit of high-performance hybrid heat production systems.

\section{References}

Barbieri, E., Y. Dai, M. Morini, M. Pinelli, P. Spina, P. Sun, and R. Wang (2014, oct). Optimal sizing of a multi-source energy plant for power heat and cooling generation. Applied Thermal Engineering 71(2), 736-750.

Di Perna, C., G. Magri, G. Giuliani, and G. Serenelli (2015). Experimental assessment and dynamic analysis of a hybrid generator composed of an air source heat pump coupled with a condensing gas boiler in a residential building. Applied Thermal Engineering 76, 86-97.

Fuentes-Cortés, L. F., J. M. Ponce-Ortega, F. Nápoles-Rivera, M. Serna-González, and M. M. El-Halwagi (2015, jul). Optimal design of integrated CHP systems for housing complexes. Energy Conversion and Management 99, 252-263.

Gimelli, A., M. Muccillo, and R. Sannino (2017, feb). Optimal design of modular cogeneration plants for hospital facilities and robustness evaluation of the results. Energy Conversion and Management 134, 20-31. 

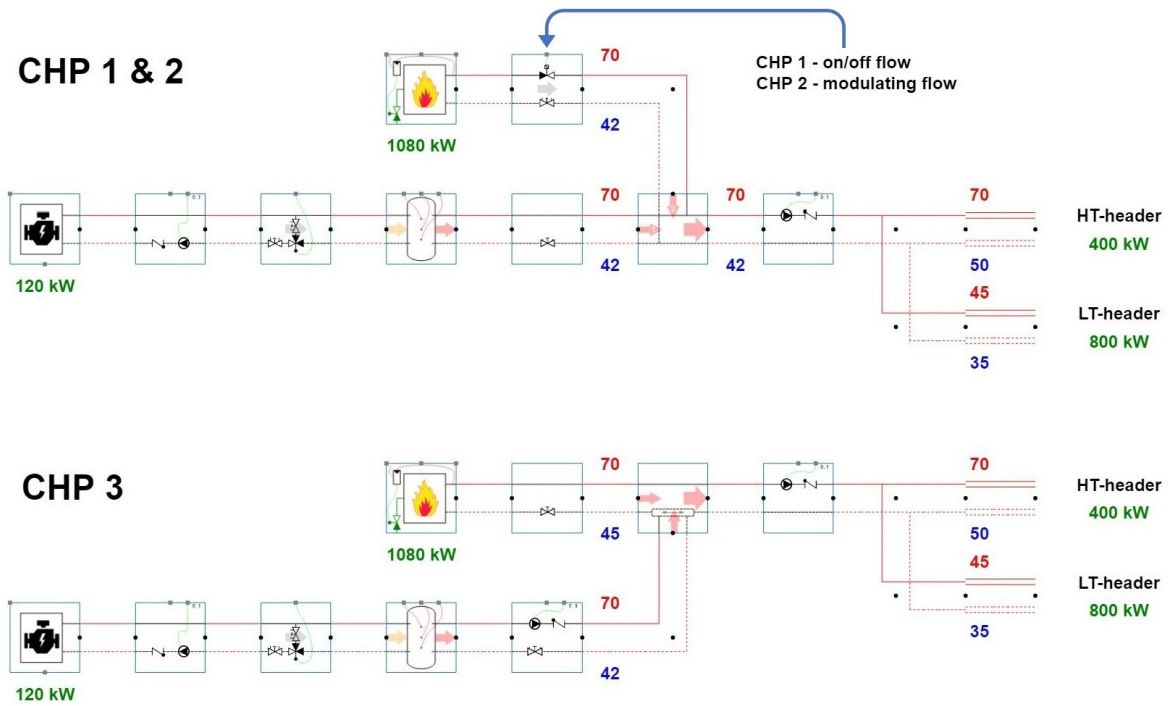

Figure 5: Hydronic concepts with an ICE-CHP as principal heat producer.

Hackel, S. and A. Pertzborn (2011). Effective design and operation of hybrid ground-source heat pumps: Three case studies. Energy and Buildings 43(12), 3497-3504

Nielsen, M. G., J. M. Morales, M. Zugno, T. E. Pedersen, and H. Madsen (2016, apr). Economic valuation of heat pumps and electric boilers in the Danish energy system. Applied Energy 167, 189200.

Ren, H., W. Gao, and Y. Ruan (2008, apr). Optimal sizing for residential CHP system. Applied Thermal Engineering 28(5-6), 514-523.

Van Riet, F., H. El Khaoui, F. Hulsbosch, G. Steenackers, and I. Verhaert (2016). Exploring the novel software Hysopt: a comparison of hydronic heat distribution systems of an apartment building. In CLIMA 2016 - proceedings of the 12th REHVA World Congress.

Van Riet, F., E. Janssen, G. Steenackers, and I. Verhaert (2019, feb). Hydronic design of cogeneration in collective residential heating systems: state-ofthe-art, comparison and improvements. Applied Thermal Engineering 148, 1246-1257.

Van Riet, F., G. Steenackers, and I. Verhaert (2018a). A new approach to model transport delay in branched pipes (in press). In 10th International Conference on System Simulation in Buildings, Volume 1, pp. 1-11.

Van Riet, F., G. Steenackers, and I. Verhaert (2018b). Design of cogeneration : a case study of an apartment block. Proceedings of the REHVA Annual Meeting Conference Low Carbon Technologies in HVAC, 23 April, 2018, Brussels, Belgium, 1-8.
Van Riet, F., G. Steenackers, and I. Verhaert (2018c). Hydronic integration of ground-coupled heat pumps in collective heating system of buildings (in press). In 10th International Conference on System Simulation in Buildings, Volume 1.

Vandebulcke, R. (2013). Hydronic Simulation and Optimisation: a simulation based study on the energy efficiency and controllability of hydronic heating systems. Ph. D. thesis, University of Antwerp.

Vandenbulcke, R., L. Mertens, and E. Janssen (2012). A simulation methodology for heat and cold distribution in thermo-hydronic networks. Building Simulation 5(3), 203-217.

Verhaert, I., R. Baetens, and F. Van Riet (2019). Performance evaluation of different micro-CHP configurations in real life conditions and the influence of part load behaviour (accepted for proceedings). In Clima 2019.

Verhaert, I., G. Mulder, and M. De Paepe (2016). Evaluation of an alkaline fuel cell system as a micro-CHP. Energy Conversion and Management 126, 434-445.

Zimny, J., P. Michalak, and K. Szczotka (2015, aug). Polish heat pump market between 2000 and 2013: European background, current state and development prospects. Renewable and Sustainable Energy Reviews 48, 791-812. 